\title{
Fragestellung der Studie
}

Rechtsherzversagen ist die häufigste Todesursache von Patienten mit PH [95]. Um eine signifikante prognostische Aussage für dieses Patientenkollektiv treffen zu können, ist eine möglichst genaue Evaluation der RV Funktion daher unerlässlich [96]. Da die CTEPH die potenziell einzig kurativ therapierbare Form der PH ist $[69,92,141]$, kommt der chirurgischen und respektive auch der interventionellen Therapie hier eine besondere Bedeutung zu. Während die PEA weiterhin als Goldstandard für operable Patienten gilt, gibt es immer auch einen relativ großen inoperablen Patientenanteil [67]. Diese Patienten können dann in Expertenzentren mittels BPA versorgt werden. Ziel dieser Arbeit ist es daher mithilfe der kardialen MRT die Effekte der BPA auf die Herzfunktion bei primär inoperablen Patienten mit CTEPH zu untersuchen. Ferner soll gezeigt werden, dass durch die Charakterisierung des Myokards mittels Mapping und insbesondere der (RV) Strainanalyse nicht invasive und im klinischen Alltag praktikable Methoden zur Verfügung stehen, um die Kontrolle von Therapieeffekten zu ermöglichen und eine prognostische Aussage hinsichtlich des Krankheitsverlaufs treffen zu können.

Elektronisches Zusatzmaterial Die elektronische Version dieses Kapitels enthält Zusatzmaterial, das berechtigten Benutzern zur Verfügung steht https://doi.org/10.1007/978-3-658-34542-6_2. 